\title{
Optimal Sensor Placement For Time Difference of Arrival Localization
}

\author{
Jason T. Isaacs, Daniel J. Klein, João P. Hespanha
}

\begin{abstract}
This paper addresses the problem of localizing a source from noisy time-of-arrival measurements. In particular, we are interested in the optimal placement of $M$ planar sensors so as to yield the best expected source location estimate. The main result, on maximizing the expected determinant of the Fisher information matrix for truncated, radially-symmetric source distributions, shows two features not previously observed. First, the sensors should be placed as far from the expected source position as possible. Second, the sensors should be arranged in a splay configuration in which neighboring sensors are separated by equal angle increments. Specific examples are given for point, uniform, and truncated-Gaussian source density functions.
\end{abstract}

\section{INTRODUCTION}

In the time-of-arrival (TOA) source localization problem, acoustic or electro-magnetic radiation emitted by a source is received by many spatially distributed sensors. Each sensor records the time at which the signal arrives and relays this information to a fusion center. Using these measurements in conjunction with the known position of each sensor, a centralized algorithm can estimate the source location and event time. Source localization has a wide range of both civilian and military applications including speaker identification in a conference room [1], [2], cell-phone geolocation [3], and sniper and mortar localization [4].

A key question that arises in the source localization problem is where to place the sensors. Answering this question amounts to finding sensor arrangements that provide the most valuable information to any particular unbiased localization algorithm. The localization algorithm performance is typically characterized by the Cramer-Rao lower bound (CRB) on the estimator variance, or by the Fisher information matrix (FIM). The CRB is the inverse of the FIM, and thus exists only when the FIM is non-singular.

A good sensor configuration results in a large FIM, or a small CRB. The size of these matrices can be measured by either the trace or the determinant. Physically, the trace of the $\mathrm{CRB}$ corresponds to the lower bound on the mean squared error of an unbiased estimate. The determinant of the CRB matrix is proportional to the lower bound on the volume of the uncertainty ellipse associated with an unbiased estimate. Here, we work with the determinant of the Fisher information matrix which is inveresly proportional to the determinant of the CRB matrix.

This material is based upon work supported by the Institute for Collaborative Biotechnologies through grant DAAD19-03-D-0004 from the U.S. Army Research Office.

Authors are with the Center for Control, Dynamical Systems, and Computation, and the Department of Electrical and Computer Engineering, University of California, Santa Barbara, CA 93106 USA. \{jtisaacs, djklein, hespanha\}eece.ucsb.edu

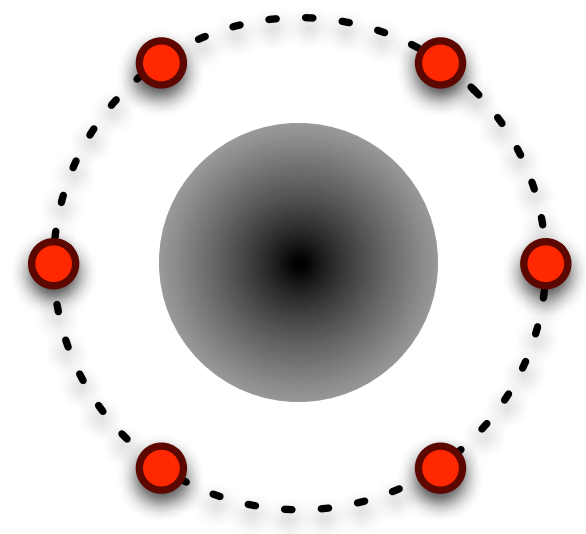

Fig. 1. A collection of six sensors is shown in an optimal configuration to localize a source distributed according to a truncated-Gaussian density function. The optimal configuration places the sensors at the boundary of the permissible region, indicated by the dashed circle, and at equal angle increments.

A majority of the previous work on optimal sensor placement considers the problem of placing sensors for a known source location. Necessary and sufficient conditions have been found for maximizing the FIM determinant [5], and sufficient conditions are known for minimizing the CRB trace [6]-[8]. In these results, the critical factor is the relative angles between the sensors. The distance between each sensor and the source is irrelevant because neither the CRB nor FIM depend on source-to-sensor distance.

Only in recent work has the optimal sensor placement problem been considered in the context of an uncertain source location. In [9], numerical optimization techniques were used to minimize the expected CRB determinant. The examples discussed suggest that optimal sensor configurations have sensors spread out along the boundary of the surveillance area. In [10], a single source is allowed to move within the surveillance area while the sensors move to optimal configurations along the boundary of the region.

We provide solutions to the optimal sensor placement problem for an uncertain source location, using the expected value of the FIM determinant as an optimality criteria. In particular, results are derived for truncated, radially symmetric source distributions when the sensors are confined to either a ring, or an annular region. Through a combination of analysis and simulation, we find two new results for the case of an unknown source location. First, the distance between the sensors and the expected source position matters and should be made as large as physically possible. Second, splay configurations like the one depicted in Fig. 1, in which 
sensors are arranged at equal angle increments, are better than other configurations suggested in the known-source literature.

This paper is organized as follows. In Section II, the problem of source localization from TDOA measurements is described. The main results concerning optimal sensor placement are presented in Section III. In Section IV, numerical results are given to support the analytical work. Optimal sensor geometries for point source, uniform, and truncatedGaussian distributions are discussed. Conclusions and future directions are discussed in Section $\mathrm{V}$.

\section{TDOA SOURCE LOCALIZATION}

In this section, we formulate the source localization problem, describe how time-of-arrival measurements are generated, and introduce the Fisher information matrix and Cramer-Rao bound. Symmetric sensor configurations are also introduced.

\section{A. The Source Localization Problem}

The main elements of a source localization problem are a single source, located at a point $\mathbf{p} \in \mathbb{R}^{2}$, and $M$ sensors located at points $\mathbf{q}:=\left[\mathbf{q}_{1}, \mathbf{q}_{2}, \ldots, \mathbf{q}_{M}\right] \in \mathbb{R}^{2 \times M}$. At an unspecified event time $t_{0}$, the source emits an acoustic or electro-magnetic signal which propagates outward at speed $\nu$. Each sensor records the time at which the signal arrives, a measurement called the time of arrival (TOA). The main objective of source localization is to infer the source location from the TOA measurements.

The noisy TOA measurement of sensor $i$ is modeled as:

$$
\hat{t}_{i}=t_{0}+\frac{d_{i}(\mathbf{p}, \mathbf{q})}{\nu}+\epsilon_{i}
$$

where

$$
d_{i}(\mathbf{p}, \mathbf{q}):=\left\|\mathbf{p}-\mathbf{q}_{i}\right\|, \quad i=1, \ldots, M
$$

is the distance between the source and the sensor. The noise, $\epsilon_{i}$, is assumed to be a zero-mean Gaussian random variable with variance $\sigma^{2}$.

The emission time $t_{0}$ can be eliminated by taking differences between TOA measurements. The resulting timedifference-of-arrival (TDOA) measurements can be written as:

$$
\hat{t}_{i j}:=\hat{t}_{i}-\hat{t}_{j}=\frac{1}{\nu} d_{i j}(\mathbf{p}, \mathbf{q})+\epsilon_{i j},
$$

where

$$
d_{i j}(\mathbf{p}, \mathbf{q}):=d_{i}(\mathbf{p}, \mathbf{q})-d_{j}(\mathbf{p}, \mathbf{q})
$$

is the difference in source-sensor distance between sensors $i$ and $j$. The noise difference, $\epsilon_{i j}:=\epsilon_{i}-\epsilon_{j}$, has variance $2 \sigma^{2}$.

A maximum of $(M-1)$ linearly independent TDOA measurements can be found for $M$ sensors. Without loss of generality, all time of arrival differences can be taken with respect to the first sensor to generate the following $(M-1) \times 1$ vectors:

$$
\begin{aligned}
& \hat{\mathbf{t}}(\mathbf{p}, \mathbf{q}):=\left[\hat{t}_{i 1}\right]_{(i=2, \ldots, M)}, \\
& \mathbf{d}(\mathbf{p}, \mathbf{q}):=\left[d_{i 1}\right]_{(i=2, \ldots, M)}, \\
& \boldsymbol{\epsilon}:=\left[\epsilon_{i 1}\right]_{(i=2, \ldots, M)} .
\end{aligned}
$$

The TDOA vector, (5), has mean

$$
\overline{\mathbf{t}}(\mathbf{p}, \mathbf{q}):=E[\hat{\mathbf{t}}]=\frac{1}{\nu} \mathbf{d}(\mathbf{p}, \mathbf{q})
$$

and the $(M-1 \times M-1)$ covariance matrix is defined as

$$
\begin{aligned}
\mathbf{Q} & :=E\left[(\hat{\mathbf{t}}-\overline{\mathbf{t}})(\hat{\mathbf{t}}-\overline{\mathbf{t}})^{T}\right] \\
& =\sigma^{2}\left[\mathbf{I}+\mathbf{1 1}^{T}\right]
\end{aligned}
$$

where $1:=\left[\begin{array}{llll}1 & 1 & \ldots & 1\end{array}\right]^{T}$.

\section{B. The Fisher Information Matrix and Cramer-Rao Bound}

The Cramer-Rao bound (CRB) provides a lower bound on the variance of an unbiased estimator and is defined as the inverse of the Fisher information matrix. The Fisher information matrix $\mathbf{F}(\mathbf{p}, \mathbf{q})$ associated with the estimation of the source location $\mathbf{p}$ is computed as [11]:

$$
\mathbf{F}(\mathbf{p}, \mathbf{q})=E\left[\left(\frac{\partial}{\partial p} \ln f(\hat{\mathbf{t}} \mid \mathbf{p})\right)\left(\frac{\partial}{\partial p} \ln f(\hat{\mathbf{t}} \mid \mathbf{p})\right)^{T}\right]
$$

where $f(\hat{\mathbf{t}} \mid \mathbf{p})$ is the PDF of $\hat{\mathbf{t}}$ given $\mathbf{p}$. Assuming the noise vector $\epsilon$ is zero mean Gaussian and independent of $\mathbf{p}$, then $f(\hat{\mathbf{t}} \mid \mathbf{p})$ is the PDF of a Gaussian random variable with mean $\overline{\mathbf{t}}(\mathbf{p})$ and covariance matrix $\mathbf{Q}$,

$$
f(\hat{\mathbf{t}} \mid \mathbf{p})=N(\hat{\mathbf{t}} ; \overline{\mathbf{t}}(\mathbf{p}), \mathbf{Q}) .
$$

This leads to the $(2 \times 2)$ Fisher information matrix given by Chan and Ho [12],

$$
\mathbf{F}(\mathbf{p}, \mathbf{q})=\frac{1}{\nu^{2}} \mathbf{G}^{T} \mathbf{Q}^{-1} \mathbf{G}
$$

where

$$
\mathbf{G}:=\left[\begin{array}{c}
\mathbf{g}_{2}^{T}-\mathbf{g}_{1}^{T} \\
\vdots \\
\mathbf{g}_{M}^{T}-\mathbf{g}_{1}^{T}
\end{array}\right]_{(M-1 \times 2)}
$$

and

$$
\mathbf{g}_{i}:=\frac{\mathbf{p}-\mathbf{q}_{i}}{\left\|\mathbf{p}-\mathbf{q}_{i}\right\|} .
$$

The Cramer-Rao lower bound is defined as the inverse of the Fisher information matrix, $\mathbf{C R}(\mathbf{p}, \mathbf{q})=\mathbf{F}(\mathbf{p}, \mathbf{q})^{-\mathbf{1}}$, and is thus defined only when $\mathbf{F}(\mathbf{p}, \mathbf{q})$ is full-rank. The matrix $\mathbf{C R}(\mathbf{p}, \mathbf{q})$ is a lower bound on the estimation error in the sense that, if $\boldsymbol{\Sigma}$ is the covariance of an unbiased estimator of $\mathbf{p}$, then $\boldsymbol{\Sigma}-\mathbf{C R}(\mathbf{p}, \mathbf{q})$ is positive semi-definite. It is important to note that the matrices $\mathbf{F}(\mathbf{p}, \mathbf{q})$ and $\mathbf{C R}(\mathbf{p}, \mathbf{q})$ depend significantly on the relative geometry of the source, $\mathbf{p}$, and the sensors, q. For the remainder of the paper, the dependence of $\mathbf{F}$ and $\mathbf{C R}$ on $\mathbf{p}$ and $\mathbf{q}$ is implicitly assumed.

\section{Known Source Optimal Sensor Placement}

Much recent work has focused on the problem of placing sensors relative to a known source location. Without loss of generality, the source can be placed at the origin. Then, denoting by $\theta_{i}$ the angle of $\mathbf{g}_{i}$, globally optimal sensor configurations for maximization of the Fisher determinant satisfy (Theorem 1 in [5]) the following two conditions: 
First moment balanced:

$$
0=\frac{1}{M} \sum_{i=1}^{M} \cos \left(\theta_{i}\right)=\frac{1}{M} \sum_{i=1}^{M} \sin \left(\theta_{i}\right) .
$$

Second moment balanced:

$$
0=\frac{1}{M} \sum_{i=1}^{M} \cos \left(2 \theta_{i}\right)=\frac{1}{M} \sum_{i=1}^{M} \sin \left(2 \theta_{i}\right) .
$$

The first moment condition is met when the sensors are spread about the source in an average sense. The second moment criteria ensures that the Fisher information matrix will have equal eigenvalues, resulting in a confidence ellipse that is circular. All configurations satisfying these conditions have a determinant of the Fisher information matrix equal to $M^{2} / 4 \sigma^{4} \nu^{4}$. Notice that when the source location is known, the determinant of the Fisher information matrix is independent of source to sensor distance.

\section{Symmetric Sensor Configurations}

When the source location is uncertain, symmetric sensor configurations play an important role.

Definition 2.1 (Symmetric Configuration): A configuration is said to be symmetric if for every $i \in\{1, \ldots, M\}$, there is an invertible mapping $f_{i}:(1, \ldots, M) \rightarrow(1, \ldots, M)$ such that

$$
\mathbf{q}_{f_{i}(j)}=\left[2 \frac{\mathbf{q}_{i} \mathbf{q}_{i}^{T}}{\left\|\mathbf{q}_{i}\right\|^{2}}-\mathbf{I}\right] \mathbf{q}_{j} .
$$

Put more simply, a configuration is symmetric when every unit vector $\mathbf{g}_{i}$ pointing from sensor $\mathbf{q}_{i}$ to the source location $\mathbf{p}$ defines an axis of reflective symmetry. Symmetric configurations need not to be second moment balanced, and vice versa second moment balanced configurations need not be symmetric, as demonstrated in Fig. 2. Another important distinction is that symmetry is distance sensitive, whereas second moment balancing only depends on relative angles.

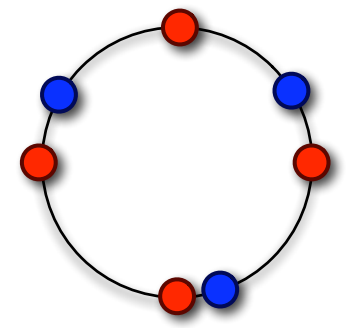

(a) Second moment balanced

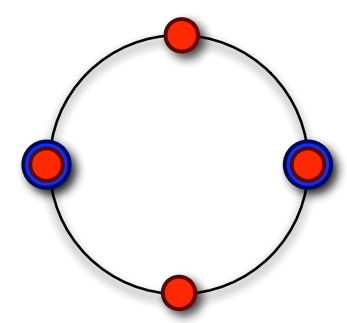

(b) Symmetric
Fig. 2. Both of these sensor configurations are first moment balanced in the sense that (15) holds, however (a) shows $M=7$ sensors in a configuration that is second moment balanced, but not symmetric whereas (b) shows $M=$ 6 sensors in a configuration that is symmetric, but not second moment balanced.

\section{Optimal Sensor Placement}

In this section, we solve the optimal sensor placement problem for truncated, radially symmetric source distributions with a given constraint on the distance from each sensor to the expected source position. At first, we require all sensors to be located at a distance $r$ from the expected source position. Later, we relax this constraint and instead allow sensors anywhere within an annulus of radii $r_{1}<r_{2}$ about the expected source position. The problem we seek to solve can be formally stated as follows.

Problem 3.1: Given $M$ sensors, a radial region $\mathcal{D} \subset \mathbb{R}^{2}$, and a radially symmetric probability density function $\phi(\|p\|)$ for the source position $\mathbf{p} \in \mathcal{P} \subset \mathbb{R}^{2}$, find the sensor locations $\mathbf{q}_{i} \in \mathcal{D}$ for $i=1, \ldots, M$ that maximize the expected value of the determinant of the Fisher information matrix,

$$
\mathbf{q}^{*}=\arg \max _{\mathbf{q} \in \mathcal{D}} \int_{\mathcal{P}} \operatorname{det}(\mathbf{F}) \phi(\|p\|) d p
$$

Without loss of generality we assume that the expected location of the source is at the origin.

Our first main result considers sensors at a fixed distance from the expected source location.

Theorem 3.2 (Fixed Distance Sensor Placement):

Assume in Problem 3.1 that the region $\mathcal{D}$ is a ring of radius $r$, centered at the origin so that

$$
\mathbf{q} \in \mathcal{D}_{r}^{M}, \mathcal{D}_{r}:=\left\{\mathbf{x} \in \mathbb{R}^{2} \mid \mathbf{x}^{T} \mathbf{x}=r^{2}\right\},
$$

and the support of the PDF is a disk with radius smaller than $r$ so that

$$
\mathbf{p} \in \mathcal{P}_{r}, \mathcal{P}_{r}:=\left\{\mathbf{x} \in \mathbb{R}^{2} \mid \mathbf{x}^{T} \mathbf{x}<r^{2}\right\} .
$$

Then, sensor configurations that are both balanced (15) and symmetric (17) satisfy the first order necessary conditions for optimality.

Before stating the proof of Theorem 3.2, we provide a lemma which allows the determinant of the Fisher information matrix to be written in a symmetric manner.

Lemma 3.3: The Fisher information matrix (12) for timeof-arrival measurement differences can be expressed as:

$$
\mathbf{F}=\frac{1}{\sigma^{2} \nu^{2}}\left(\sum_{i=1}^{M} \mathbf{g}_{i} \mathbf{g}_{i}^{T}-M \overline{\mathbf{g}} \overline{\mathbf{g}}^{T}\right),
$$

and the determinant of (21) is given by

$$
\begin{aligned}
\operatorname{det}(\mathbf{F})=\frac{1}{\sigma^{4} \nu^{4}} & {\left[\left(\sum_{i=1}^{M} g_{i, x}^{2}-M \bar{g}_{x}^{2}\right)\left(\sum_{i=1}^{M} g_{i, y}^{2}-M \bar{g}_{y}^{2}\right)\right.} \\
- & \left.\left(\sum_{i=1}^{M} g_{i, x} g_{i, y}-M \bar{g}_{x} \bar{g}_{y}\right)^{2}\right],
\end{aligned}
$$

where $\overline{\mathrm{g}}$, defined by

$$
\overline{\mathrm{g}}:=\frac{1}{M} \sum_{i=1}^{M} \mathbf{g}_{i},
$$

is the average of the $\mathrm{g}_{i}$ vectors. A proof of Lemma 3.3 is available in the Appendix.

We are now ready to give the proof of the first theorem. Proof: (of Theorem 3.2) We begin by forming the Lagrangian 
of (18),

$$
L(\mathbf{q}, \boldsymbol{\mu}):=\int_{\mathcal{P}_{r}} \operatorname{det}(\mathbf{F}) \phi(\|p\|) d p+\sum_{i=1}^{M} \mu_{i}\left(\mathbf{q}_{i}^{T} \mathbf{q}_{i}-r^{2}\right),
$$

where $\boldsymbol{\mu} \in \mathbb{R}^{M}$ is a vector of Lagrange multipliers. A point $\mathbf{q}^{*}$ satisfies the Karush-Kuhn-Tucker (KKT) [13] first order necessary optimality conditions for this Lagrangian if there exists a vector $\boldsymbol{\mu}^{*} \in \mathbb{R}^{M}$ such that:

$$
\begin{aligned}
& 0=\mathbf{q}_{i}^{* T} \mathbf{q}_{i}^{*}-r^{2} \quad \forall i \in\{1,2, \ldots, M\} \\
& \mathbf{0}=\boldsymbol{\nabla}\left[\int_{\mathcal{P}_{r}} \operatorname{det}(\mathbf{F}) \phi(\|p\|) d p+\sum_{j=1}^{M} \mu_{j}^{*}\left(\mathbf{q}_{j}^{* T} \mathbf{q}_{j}^{*}-r^{2}\right)\right]
\end{aligned}
$$

where $\nabla$ denotes the differential operator

$$
\boldsymbol{\nabla}:=\left[\frac{\partial}{\partial \mathbf{q}_{i}}\right]_{(i=1, \ldots, M)} .
$$

Condition (26) can be written for each $i$ as:

$$
2 \mu_{i} \mathbf{q}_{i}=-\int_{\mathcal{P}_{r}} \frac{\partial \operatorname{det}(\mathbf{F})}{\partial \mathbf{q}_{i}} \phi(\|p\|) d p .
$$

After some algebra, we find using Lemma 3.3 that

$$
\frac{\partial \operatorname{det} \mathbf{F}}{\partial \mathbf{q}_{i}}=\frac{2}{\left\|\mathbf{p}-\mathbf{q}_{i}\right\|} \mathbf{g}_{i}^{T} \mathbf{F}\left(\mathbf{g}_{i}-\overline{\mathbf{g}}\right)^{\perp} \mathbf{g}_{i}^{\perp},
$$

where the perpendicular operator $\perp$ is defined by

$$
\mathbf{x}^{\perp}=\left[\begin{array}{cc}
0 & -1 \\
1 & 0
\end{array}\right] \mathbf{x}, \quad \forall \mathbf{x} \in \mathbb{R}^{2} .
$$

Symmetric configurations, from Definition 2.1, play an important role in the integral in (28). To see the symmetry, consider a coordinate rotation in which the $i^{t h}$ sensor, $\mathbf{q}_{i}$, is placed on the positive $x$-axis. Then take two source points, $\mathbf{p}$ and $\tilde{\mathbf{p}}$, for which $\tilde{\mathbf{p}}$ is $\mathbf{p}$ mirrored about the $x$-axis,

$$
\tilde{\mathbf{p}}=\mathbf{R} \mathbf{p},
$$

where $\mathbf{R}$ is the elementary reflector about the $\mathrm{x}$-axis defined as:

$$
\mathbf{R}:=\left[\begin{array}{cc}
1 & 0 \\
0 & -1
\end{array}\right] .
$$

Some algebra reveals

$$
\left.\frac{\partial \operatorname{det} \mathbf{F}}{\partial \mathbf{q}_{i}}\right|_{(\tilde{\mathbf{p}}, \mathbf{q})}=\left.\mathbf{R} \frac{\partial \operatorname{det} \mathbf{F}}{\partial \mathbf{q}_{i}}\right|_{(\mathbf{p}, \mathbf{q})} .
$$

To show the optimality of the $i^{\text {th }}$ sensor (which now lies on the $x$-axis), we employ (31) to split the integral in (28) into two parts. When integrating over points not on the $\mathrm{x}$ axis, for every $\mathbf{p}$ there exists a $\tilde{\mathbf{p}}$ for which the sum of the two integrands have equal and opposite $y$-components and equal $x$-components.

$$
\begin{aligned}
\frac{\partial}{\partial \mathbf{q}_{i}} \int_{\mathcal{P}_{r}} \operatorname{det} \mathbf{F} \phi(\|p\|) d p & =\int_{\mathcal{P}_{0}} \frac{\partial \operatorname{det} \mathbf{F}}{\partial \mathbf{q}_{i}} \phi(\|p\|) d p \\
& +\int_{\mathcal{P}_{+}}[\mathbf{I}+\mathbf{R}] \frac{\partial \operatorname{det} \mathbf{F}}{\partial \mathbf{q}_{i}} \phi(\|p\|) d p .
\end{aligned}
$$

In the first part, integrated over $\mathcal{P}_{0} \subset \mathcal{P}_{r}, \mathbf{p}$ lies on the $x$ axis and in the second part, integrated over $\mathcal{P}_{+} \subset \mathcal{P}_{r}$, $\mathbf{p}$ has a positive $y$-component. Integrating over $\mathcal{P}_{0}$ is trivially zero because the integrand is zero at all points $\mathbf{p} \in \mathcal{P}_{0}$ due to the symmetry of the configuration. Notice that in (34) the $y$-component cancels because

$$
\mathbf{I}+\mathbf{R}=\left[\begin{array}{ll}
2 & 0 \\
0 & 0
\end{array}\right]
$$

indicating that the right hand side of (28) is aligned with $\mathbf{q}_{i}$. Thus, we conclude that there exists a multiplier,

$$
\mu_{i}=-\int_{\mathcal{P}_{+}} \frac{\mathbf{q}_{i}^{T}}{\left\|\mathbf{q}_{i}\right\|^{2}}\left[\begin{array}{ll}
1 & 0 \\
0 & 0
\end{array}\right] \frac{\partial \operatorname{det} \mathbf{F}}{\partial \mathbf{q}_{i}} \phi(\|p\|) d p
$$

for each $i$ so that the optimality conditions are satisfied.

We next consider a generalization of Theorem 3.2 in which the sensors are confined to an annulus of radii $r_{1}<r_{2}$ instead of an $r$-radius ring, but first we introduce a particular symmetric configuration that will be used throughout the remainder of this paper.

Definition 3.4 (Splay Configuration): A symmetric configuration is said to be splay if

$$
\theta_{j}-\theta_{i}=\frac{2 \pi}{N}, \text { for each } j=i+1,
$$

where $\theta_{i}$ is the angle of the vector $\mathbf{q}_{i}$ with respect to the $\mathrm{x}$-axis.

Theorem 3.5 (Variable Distance Sensor Placement):

Assume in Problem 3.1 that the region $D$ is an annulus of radii $r_{1}<r_{2}$, centered at the origin,

$$
\mathbf{q} \in D_{a}^{M}, D_{a}:=\left\{\mathbf{x} \in \mathbb{R}^{2} \mid r_{1}^{2} \leq \mathbf{x}^{T} \mathbf{x} \leq r_{2}^{2}\right\},
$$

and the probability density function is truncated at a radius less than $r_{1}$ so that

$$
\mathbf{p} \in \mathcal{P}_{a}, \mathcal{P}_{a}:=\left\{\mathbf{x} \in \mathbb{R}^{2} \mid \mathbf{x}^{T} \mathbf{x}<r_{1}^{2}\right\} .
$$

Then, sensor configurations on the outer boundary of the annulus,

$$
\mathbf{q}_{i}^{T} \mathbf{q}_{i}=r_{2}^{2}, \quad \forall i \in\{1,2, \ldots, M\},
$$

that are additionally splay (37) satisfy the first order necessary optimality conditions.

Proof: (of Theorem 3.5) The Lagrangian for (18) with the inequality constraints (38) is now

$$
\begin{aligned}
L(\mathbf{q}, \boldsymbol{\mu}, \boldsymbol{\lambda}):=\int_{\mathcal{P}_{a}} \operatorname{det}(\mathbf{F}) & \phi(\|p\|) d p+\sum_{i=1}^{M} \mu_{i}\left(r_{1}^{2}-\mathbf{q}_{i}^{T} \mathbf{q}_{i}\right) \\
& +\sum_{i=1}^{M} \lambda_{i}\left(\mathbf{q}_{i}^{T} \mathbf{q}_{i}-r_{2}^{2}\right)
\end{aligned}
$$

where $\boldsymbol{\mu} \in \mathbb{R}^{M}$ and $\boldsymbol{\lambda} \in \mathbb{R}^{M}$, are vectors of Lagrange multipliers. The KKT first order necessary conditions for 


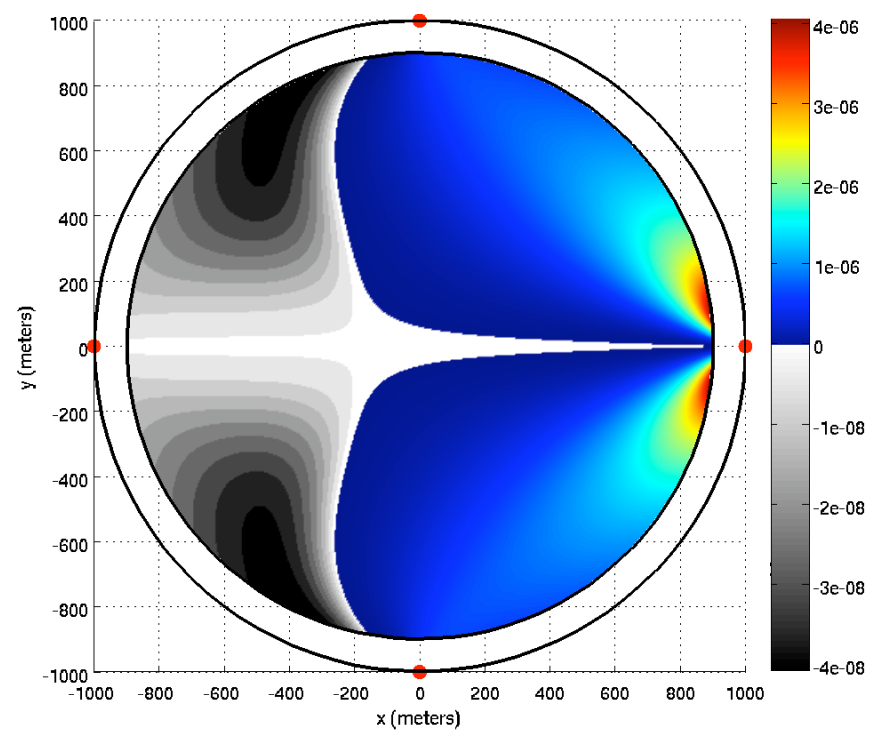

Fig. 3. The integrand of (50) is plotted for $\mathbf{p}$ inside a disk of radius $r_{1}=900$ with $M=4$ sensors placed in a splay configuration at radius $r_{2}=1000$. The important thing to note here is that the integral will be positive even though the integrand is negative for some p. A nonlinear scaling has been applied to the colormap to show details for negative values of the integrand.

optimality are now $\forall i \in\{1,2, \ldots, M\}$ :

$$
\begin{aligned}
& 0 \geq r_{1}^{2}-\mathbf{q}_{i}^{* T} \mathbf{q}_{i}^{*} \\
& 0 \leq \mu_{i}^{*} \\
& 0=\mu_{i}^{*}\left(r_{1}^{2}-\mathbf{q}_{i}^{* T} \mathbf{q}_{i}^{*}\right) \\
& 0 \geq \mathbf{q}_{i}^{* T} \mathbf{q}_{i}^{*}-r_{2}^{2} \\
& 0 \leq \lambda_{i}^{*} \\
& 0=\lambda_{i}^{*}\left(\mathbf{q}_{i}^{* T} \mathbf{q}_{i}^{*}-r_{2}^{2}\right) \\
& \mathbf{0}=\boldsymbol{\nabla}(\mathbf{q}, \boldsymbol{\mu}, \boldsymbol{\lambda}) .
\end{aligned}
$$

Here, the first six relationships are complementary slackness conditions to ensure that sensors remain in $\mathcal{D}_{a}$. Because the sensor configuration in which we are interested have all sensors at the outer radius $r_{2}$ of $\mathcal{D}_{a}(40), \mu_{i}=0, \forall i \in$ $1, \ldots, M$, the relationship (48) is identical to (26) from the fixed radius problem. Thus we have already shown that symmetric, and hence splay, configurations satisfy (48) in the proof of Theorem 3.2.

What remains to be shown is that all $M$ Lagrange multipliers, $\lambda_{i}, i=1, \ldots, M$ have positive sign (46). Using (29), (48) becomes

$$
\mathbf{0}=\int_{\mathcal{P}_{a}} \frac{2}{\left\|\mathbf{p}-\mathbf{q}_{i}\right\|} \mathbf{g}_{i}^{T} \mathbf{F}\left(\mathbf{g}_{i}-\overline{\mathbf{g}}\right)^{\perp} \mathbf{g}_{i}^{\perp} \phi(\|p\|) d p+2 \lambda_{i} \mathbf{q}_{i} .
$$

To show that each Lagrange multiplier is positive, we solve for $\lambda_{i}$ and write the integration in polar coordinates,

$\lambda_{i}=\int_{0}^{r_{1}} \int_{0}^{2 \pi} \frac{-1}{\left\|\mathbf{p}-\mathbf{q}_{i}\right\|\left\|\mathbf{q}_{i}\right\|^{2}} \mathbf{g}_{i}^{T} \mathbf{F}\left(\mathbf{g}_{i}-\overline{\mathbf{g}}\right)^{\perp} \mathbf{q}_{i}^{T} \mathbf{g}_{i}^{\perp} d p_{\theta} \phi(\rho) \rho d \rho$,

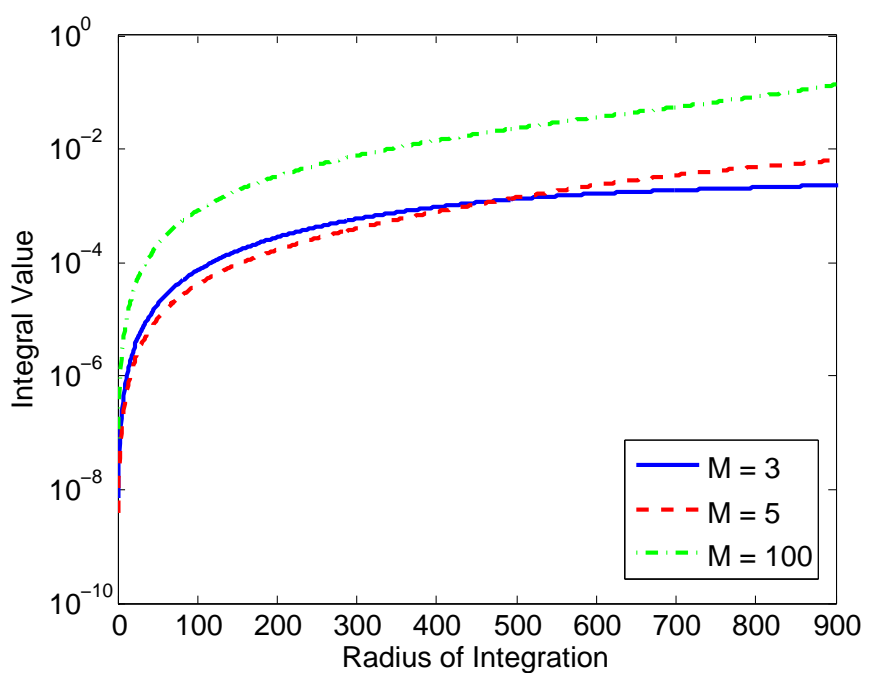

Fig. 4. The inner integral in (50) is plotted vs the radius $\rho$ of $\mathbf{p}$ for $M$ sensors placed in a splay configuration at a radius $r_{2}=1000$. For all $\rho$, the inner integral evaluates to a positive number indicating that $\lambda_{i}$ is positive. As $\rho$ approaches zero the distibution looks more like a point mass, thus the inner integral approaches zero.

where $\mathbf{p}=\rho \angle p_{\theta}$. Because the radially symmetric density function $\phi(\rho)$ is non-negative, we need only to show that the inner integral is positive for every $\rho$. Unfortunately, the integrand in (50) does not have constant sign, see Fig. 3 for example. Because a closed form computation of the integral appears to be intractable, we refer the reader to Fig. 4, which shows the inner integral plotted against $\rho$ for various $M$ in a splay configuration.

\section{Numerical Results}

In this section we include two numerical results that support Theorem 3.5 and motivate a conjecture that is left for future research. In the first, we study the splay configuration found in Theorem 3.5 and evaluate how the expected Fisher determinant varies with the outer radius $r_{2}$. In the second, we evaluate the expected Fisher determinant for a subset of configurations found in Theorem 3.2.

\section{A. Expected Fisher Determinant Maximum at the Boundary}

In Theorem 3.5 we have shown that if the region confining the sensors $\mathcal{D}_{a}$ is an annulus of radius $r_{1}<r_{2}$, centered at the expected location of the source, then sensors placed at the boundary of the region $\mathcal{D}_{a}$ in a splay configuration meet the first order necessary conditions for optimality. The purpose of this section is to examine how the expected Fisher determinant changes with the outer radius of the boundary. In Fig. 5 it can be seen that the expected Fisher determinant increases monontonically with $r_{2}$ for both uniform and truncated-Gaussian distributions. This observation supports Theorem 3.5 in that if sensors are in a splay configuration about the expected source location, then it is beneficial for all the sensors to move out radially. 


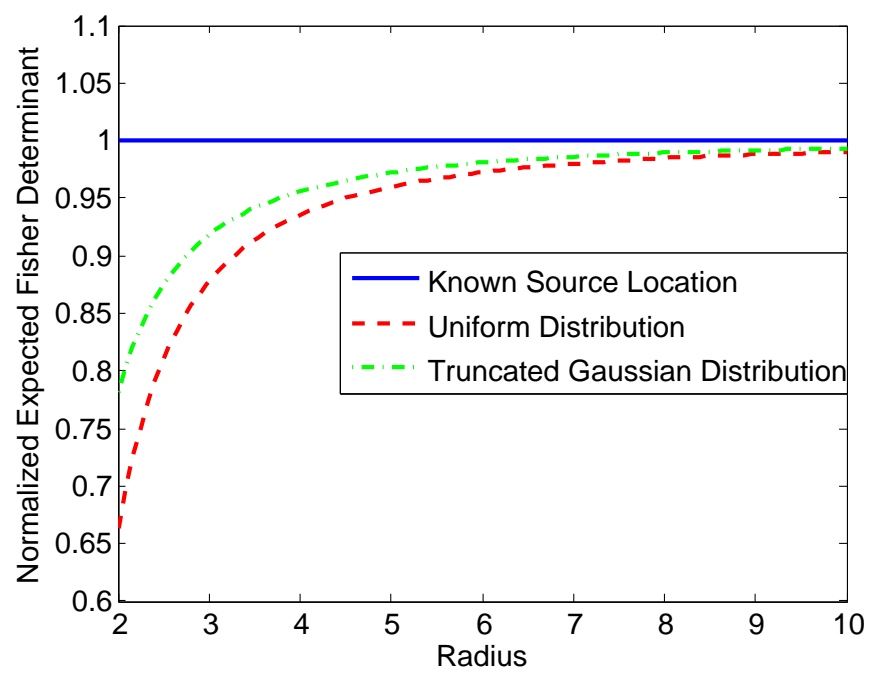

Fig. 5. The expected value of the Fisher information determinant as a function of outer radius $r_{2}$ of region $\mathcal{D}_{a}$ for $M=6$ sensors in splay configuration.

\section{B. Splay Configuration Maximizes Fisher Determinant on Ring}

The result of Theorem 3.2 states that any balanced and symmetric configuration meets the necessary conditions of first order optimality. However, the configurations with sensors at equal angle increments seem to yield higher expected Fisher determinants than other configurations satisfying the balancing and symmetry conditions.

Conjecture 4.1 (Splay Conjecture): Of all the balanced and symmetric configurations satisfying the first order necessary conditions in Theorem 3.2, splay configurations, from Definition 3.4, give the largest expected Fisher determinant.

Consider the case where $M=6$, starting in the splay configuration for which (15), (16), and (37) are all satisfied. If we hold the position of three of the even ordered sensors constant while rotating the odd ordered three sensors about the origin by $\alpha \in\left[\begin{array}{ll}0 & 4 \pi / M\end{array}\right]$, then (15) and (16) remain satisfied, but the symmetry conditions (17) are only satisfied when $\alpha=0, \alpha=2 \pi / M$, and $\alpha=4 \pi / M$. This rotation by $\alpha$ takes the configurations from an $M=6$ splay configuration through the point where there are two sensors at each location of a $M=3$ splay configuration and back to a $M=6$ splay configuration again. All of these configurations meet the optimality conditions for a known source at the origin [?], but only $\alpha=0, \alpha=2 \pi / M$, and $\alpha=4 \pi / M$ satisfy the symmetry conditions required by Theorem 3.2. The results in Fig. 6 show that the splay configuration at $\alpha=0$ and $\alpha=4 \pi / M$ achieve the maximum expected determinant of FIM for both uniform and truncated-Gaussian distributions while the repeated configuration at $\alpha=2 \pi / M$ does not.

\section{CONCLusions}

The work in this paper has addressed the optimal sensor placement problem considering a source node distributed according to a truncated radially-symmetric probability density function. The results suggest that good sensor configurations

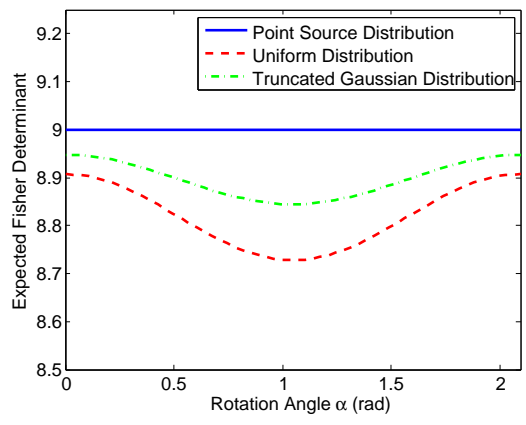

(a) Expected Fisher Determinant

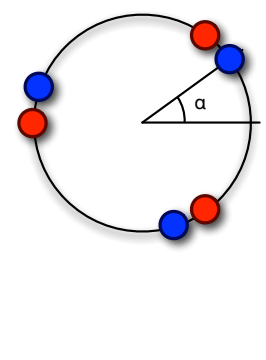

(b) $M=6$ Sensors

Fig. 6. In (a) the expected value of the Fisher information determinant is plotted as a function of rotation angle $\alpha$ between two sets of $M=6$ first order optimal configurations. When $\alpha=2 \pi / M$ the sensors are arranged in a configuration of duplicate sensors in a $M=3$ splay configuration which satifies the first order necessary conditions in Theorem 3.5, but does not maximize the expected Fisher determinant. In (b) the $M=3$ blue sensors are shown rotated by angle $\alpha$ while the $M=3$ red sensors are held stationary.

are both balanced and symmetric. Overall, the results show that the distance between the source and the sensor is influential, and that splay configurations are better than other ones. These results are new and were not apparent in previous work that considered a known source location.

There are many directions in which this work could be extended. First, both Theorems 3.2 and 3.5 should be extended to include any radially symmetric source distribution that may extend outside the area where sensors may be placed. Also, further analytical results could be provided regarding the advantage of splay states over other symmetric and balanced states.

In another direction, the radial symmetry of the source distribution should be relaxed. In an urban environment, the source distribution is unlikely to be radially symmetric. Further, sensor constraints other than a ring or annulus could be considered.

\section{APPENDIX}

\section{A. Proof of Lemma 3.3}

Proof: (of Lemma 3.3) By induction, beginning with (12) for $M=3$,

$$
\begin{aligned}
\mathbf{F}_{3} & =\frac{1}{\nu^{2}} \mathbf{G}^{T} \mathbf{Q}^{-1} \mathbf{G} \\
& =\frac{1}{\sigma^{2} \nu^{2}}\left[\begin{array}{ll}
\mathbf{g}_{2}-\mathbf{g}_{1} & \mathbf{g}_{3}-\mathbf{g}_{1}
\end{array}\right]\left[\mathbf{I}-\frac{1}{3} \mathbf{1} \mathbf{1}^{T}\right]\left[\begin{array}{l}
\mathbf{g}_{2}^{T}-\mathbf{g}_{1}^{T} \\
\mathbf{g}_{3}^{T}-\mathbf{g}_{1}^{T}
\end{array}\right] \\
& =\frac{1}{\sigma^{2} \nu^{2}}\left(\sum_{i=1}^{3} \mathbf{g}_{i} \mathbf{g}_{i}^{T}-3\left(\frac{1}{3}\right) \sum_{i=1}^{3} \mathbf{g}_{i}\left(\frac{1}{3}\right) \sum_{j=1}^{3} \mathbf{g}_{j}^{T}\right) \\
& =\frac{1}{\sigma^{2} \nu^{2}}\left(\sum_{i=1}^{3} \mathbf{g}_{i} \mathbf{g}_{i}^{T}-3 \overline{\mathbf{g}} \overline{\mathbf{g}}^{T}\right)
\end{aligned}
$$


Assuming the lemma holds for $M$,

$$
\begin{aligned}
\mathbf{F}_{M} & =\frac{1}{\nu^{2}} \mathbf{G}_{M}^{T} \mathbf{Q}_{M}^{-1} \mathbf{G}_{M} \\
& =\frac{1}{\sigma^{2} \nu^{2}}\left(\sum_{i=1}^{M} \mathbf{g}_{i} \mathbf{g}_{i}^{T}-M \overline{\mathbf{g}} \overline{\mathbf{g}}^{T}\right),
\end{aligned}
$$

we examine $M+1$ letting $\Gamma=\frac{1}{M+1}$ and dropping $\nu$ and $\sigma$ for brevity,

$$
\begin{aligned}
& \mathbf{Q}_{M+1}^{-1}=\left[\begin{array}{cc}
\mathbf{Q}_{M}^{-1}+\left(\frac{\Gamma}{M}\right) \mathbf{1}_{M-1} \mathbf{1}_{M-1}^{T} & -\Gamma \mathbf{1}_{M-1} \\
-\Gamma \mathbf{1}_{M-1}^{T} & \Gamma M
\end{array}\right] \\
& \mathbf{F}_{M+1}=\left[\begin{array}{ll}
\mathbf{G}_{M}^{T} & \mathbf{g}_{M+1}-\mathbf{g}_{1}
\end{array}\right] \mathbf{Q}_{M+1}^{-1}\left[\begin{array}{c}
\mathbf{G}_{M} \\
\mathbf{g}_{M+1}^{T}-\mathbf{g}_{1}^{T}
\end{array}\right] \\
& =\mathbf{F}_{M}+\frac{\Gamma}{M} \mathbf{G}_{M}^{T} \mathbf{1}_{M-1} \mathbf{1}_{M-1}^{T} \mathbf{G}_{M} \\
& -\Gamma \mathbf{G}_{M}^{T} \mathbf{1}_{M-1}\left(\mathbf{g}_{M+1}^{T}-\mathbf{g}_{1}^{T}\right) \\
& -\Gamma\left(\mathbf{g}_{M+1}-\mathbf{g}_{1}\right) \mathbf{1}_{M-1}^{T} \mathbf{G}_{M} \\
& +\Gamma M\left(\mathbf{g}_{M+1}-\mathbf{g}_{1}\right)\left(\mathbf{g}_{M+1}^{T}-\mathbf{g}_{1}^{T}\right) \\
& =\mathbf{F}_{M}+\Gamma M\left(\overline{\mathbf{g}}_{M}-\mathbf{g}_{M+1}\right)\left(\overline{\mathbf{g}}_{M}-\mathbf{g}_{M+1}\right)^{T} \\
& =\sum_{i=1}^{M} \mathbf{g}_{i} \mathbf{g}_{i}^{T}+\mathbf{g}_{M+1} \mathbf{g}_{M+1}^{T}-\Gamma\left[M^{2} \overline{\mathbf{g}}_{M} \overline{\mathbf{g}}_{M}^{T}\right. \\
& \left.+\mathbf{g}_{M+1} \mathbf{g}_{M+1}^{T}+M \overline{\mathbf{g}}_{M} \mathbf{g}_{M+1}^{T}+M \mathbf{g}_{M+1} \overline{\mathbf{g}}_{M}^{T}\right] \\
& =\sum_{i=1}^{M+1} \mathbf{g}_{i} \mathbf{g}_{i}^{T} \\
& -\Gamma\left[\left(M \overline{\mathbf{g}}_{M}+\mathbf{g}_{M+1}\right)\left(M \overline{\mathbf{g}}_{M}+\mathbf{g}_{M+1}\right)^{T}\right] \\
& =\sum_{i=1}^{M+1} \mathbf{g}_{i} \mathbf{g}_{i}^{T}-(M+1)\left[\overline{\mathbf{g}}_{M+1} \overline{\mathbf{g}}_{M+1}^{T}\right] .
\end{aligned}
$$

The determinant of the Fisher information matrix (22) follows directly from the symmetric form of the Fisher information matrix (21),

$$
\operatorname{det}(\mathbf{F})=\frac{1}{\sigma^{4} \nu^{4}} \operatorname{det}\left(\sum_{i=1}^{M} \mathbf{g}_{i} \mathbf{g}_{i}^{T}-M \overline{\mathbf{g}} \overline{\mathbf{g}}^{T}\right) .
$$

\section{REFERENCES}

[1] Y. Huang, J. Benesty, and G. W. Elko, "Passive acoustic source localization for video camera steering," in Proceedings of the 2000 IEEE International Conference on Acoustics, Speech, and Signal Processing, vol. 2, 2000, pp. II909-II912 vol.2.

[2] W.-K. Ma, B. Vo, S. S. Singh, and A. Baddeley, "Tracking an unknown time-varying number of speakers using TDOA measurements: A random finite set approach," IEEE Transactions on Signal Processing, vol. 54, no. 9, pp. 3291-3304, Sept. 2006.

[3] J. J. Caffery and G. L. Stuber, "Overview of radiolocation in CDMA cellular systems," IEEE Communications Magazine, vol. 36, no. 4, pp. 38-45, Apr. 1998.

[4] G. Simon, M. Maróti, A. Lédeczi, G. Balogh, B. Kusy, A. Nádas, G. Pap, J. Sallai, and K. Frampton, "Sensor network-based countersniper system," Proceedings of the Second ACM International Conference on Embedded Networked Sensor Systems, pp. 1-12, 2004.

[5] A. N. Bishop, B. Fidan, B. D. O. Anderson, P. N. Pathirana, and K. Dogancay, "Optimality analysis of sensor-target geometries in passive localization: Part 2 - time-of-arrival based localization," Proceedings of the 3rd International Conference on Intelligent Sensors, Sensor Networks and Information, pp. 13-18, Dec. 2007.
[6] B. Yang and J. Scheuing, "Cramer-Rao bound and optimum sensor array for source localization from time differences of arrival," Proceedings of the 2005 IEEE International Conference on Acoustics, Speech, and Signal Processing, vol. 4, pp. iv/961-iv/964 Vol. 4, Mar. 2005.

[7] — - "A theoretical analysis of 2D sensor arrays for TDOA based localization," Proceedings of the 2006 IEEE International Conference on Acoustics, Speech, and Signal Processing, vol. 4, pp. IV-IV, May 2006.

[8] B. Yang, "Different sensor placement strategies for TDOA based localization," Proceedings of the 2007 IEEE International Conference on Acoustics, Speech, and Signal Processing, vol. 2, pp. II-1093-II1096, Apr. 2007.

[9] J. Neering, M. Bordier, and N. Maizi, "Optimal passive source localization," Proceedings of the 2007 International Conference on Sensor Technologies and Applications, pp. 295-300, Oct. 2007.

[10] S. Martínez and F. Bullo, "Optimal sensor placement and motion coordination for target tracking," Automatica, vol. 42, no. 4, pp. 661 - 668, 2006.

[11] J. M. Mendel, Lessons in Estimation Theory for Signal Processing, Communications, and Control. New Jersey: Prentice-Hall, 1995.

[12] Y. T. Chan and K. C. Ho, "A simple and efficient estimator for hyperbolic location," IEEE Transactions on Signal Processing, vol. 42, no. 8, pp. 1905-1915, Aug. 1994.

[13] S. Boyd and L. Vandenberghe, Convex Optimization. Cambridge University Press, Mar. 2004. 